\title{
Existence of positive solution for fourth order superlinear singular semipositone differential system
}

\author{
Wang Feng ${ }^{1}$, Junmin Zhang ${ }^{2}$ \\ ${ }^{1}$ Jiangsu Union Technical Institute, Yancheng College of Mechatronic Technology, Yancheng, Jiangsu 224005, China \\ ${ }^{2}$ Department of Mathmatics and Applied Mathmatics, Hanshan Normal University, Chaozhou, Guangdong 521041, China
}

\section{Email address:}

wfsxjd@163.com (W. Feng),gd_zjm@126.com (J.M. Zhang)

\section{To cite this article:}

Wang Feng, Junmin Zhang. Existence of Positive Solution for Fourth Order Superlinear Singular Semipositone Differential System. Pure and Applied Mathematics Journal. Vol. 2, No. 6, 2013, pp. 179-183. doi: 10.11648/j.pamj.20130206.12

\begin{abstract}
By transforming the boundary value problem into the corresponding fixed-point problem of a completely continuous operator, the existence is obtained in the paper for two-point boundary value problem of fourth order superlinear singular semipositone differential system via the fixed point theorem concerning cone compression and expansion in norm type.
\end{abstract}

Keywords: Superlinear Singular Semipositone Differential System, Positive Solution, Fixed Point Theorem, Cone

\section{Introduction}

In this paper, we study the following superlinear fourth order singular semipositone differential system

$$
\left\{\begin{array}{l}
x^{(4)}(t)=\lambda f(t, x(t), y(t))+p(t), \\
y^{(4)}(t)=\lambda g(t, x(t), y(t))+q(t), \quad t \in(0,1),
\end{array}\right.
$$

subject to the boundary conditions

$$
\left\{\begin{array}{l}
x(0)=x(1)=x^{\prime \prime}(0)=x^{\prime \prime}(1)=0, \\
y(0)=y(1)=y^{\prime \prime}(0)=y^{\prime \prime}(1)=0
\end{array}\right.
$$

where

$1 \leq \lambda<2, f$ and $g:(0,1) \times[0,+\infty) \times[0,+\infty) \rightarrow[0,+\infty)$ are continuous and may be singular at $t=0,1 . p$ and $q:(0,1)$

$\rightarrow(-\infty,+\infty)$ are Lebesgue integrable.

The method is fixed point theorem concerning cone compression and expansion. For the concepts and property of fixed point index theory we refer to [1].

Recently, the existence of positive solutions for nonlinear singular semipositone boundary value problems has been studied extensively, see [2-6]. However, the results are seldom for fourth order superlinear singular semipositone differential system boundary value problem. In the previous literature, a great deal of work was devoted to the case that $f$ and $g$ are nonnegative and have no singularities or $p(t) \equiv 0, q(t) \equiv 0$, see $[7,8]$. When $f$ and $g$ are allowed to change sign, singular and $p(t) \equiv 0, q(t) \equiv 0,[9]$ obtained the existence of positive solutions for nonlinear three-point boundary value problems. Under the given conditions that $f$ and $g$ are nonnegative, singular and $p(t), q(t)$ change sign, [10] obtained the existence of positive solutions for two-point boundary value problems. Motivated by $[6,10]$, we study the existence of positive solutions for the twopoint boundary value problem of fourth order superlinear singular semipositone differential system by using the fixed point theorem concerning cone compression and expansion in norm type in the paper.

\section{Preliminaries}

Let $X=C([0,1] ; R) \times C([0,1] ; R)$ be a real Banach space equipped with for any $(u, v) \in X$,

$$
\|(u, v)\|=\|u\|+\|v\|, \quad\|u\|=\max _{t \in[0,1]}|u(t)|, \quad\|v\|=\max _{t \in[0,1]}|v(t)|,
$$

where $R$ is a real number set.

Let us define a cone $P$ of $X$ by

$P=\{(x, y) \in X \mid x(t) \geq t(1-t)\|x\|, y(t) \geq t(1-t)\|y\|, \forall t \in[0,1]\}$

Next we introduce Green's function to $u^{\prime \prime}(0)=0$ subject to boundary condition $u(0)=u(1)=0$,

$$
G(t, s)= \begin{cases}s(1-t), & 0 \leq s \leq t \leq 1 \\ t(1-s), & 0 \leq t \leq s \leq 1 .\end{cases}
$$


For any $y \in C[0,1]$, let us define a funtion by

$$
[y(t)]^{*}= \begin{cases}y(t), & y(t) \geq 0, \\ 0, & y(t)<0 .\end{cases}
$$

For the convenience, in this paper we make the following assumptions:

$\left(H_{1}\right)$ There exist constant $\lambda_{i}>\mu_{i}>1,(i=1,2,3,4)$ such that, $\forall(t, x, y) \in(0,1) \times[0,+\infty) \times[0,+\infty)$, and $c \in(0,1)$,

$$
\begin{aligned}
& c^{\lambda_{1}} f(t, x, y) \leq f(t, c x, y) \leq c^{\mu_{1}} f(t, x, y), \\
& c^{\lambda_{2}} f(t, x, y) \leq f(t, x, c y) \leq c^{\mu_{2}} f(t, x, y), \\
& c^{\lambda_{3}} g(t, x, y) \leq g(t, c x, y) \leq c^{\mu_{3}} g(t, x, y), \\
& c^{\lambda_{4}} g(t, x, y) \leq g(t, x, c y) \leq c^{\mu_{4}} g(t, x, y) .
\end{aligned}
$$

$\left(\mathrm{H}_{2}\right)$

$$
\begin{gathered}
\int_{0}^{1} p_{-}(s) \mathrm{d} s=r_{1}>0, \quad \int_{0}^{1} q_{-}(s) \mathrm{d} s=r_{3}>0, \\
\int_{0}^{1} G(s, s)\left[f(s, 1,1)+p_{+}(s)\right] \mathrm{d} s=r_{2}<\frac{1}{\lambda}, \\
\int_{0}^{1} G(s, s)\left[g(s, 1,1)+q_{+}(s)\right] \mathrm{d} s=r_{4}<\frac{1}{\lambda}, \\
f(t, 0,1)>0, \quad f(t, 1,0)>0, \quad g(t, 0,1)>0, \\
g(t, 1,0)>0, \quad \forall t \in(0,1) .
\end{gathered}
$$

$\int_{0}^{1} G(s, s) f(s, 1,1) \mathrm{d} s$ and $\int_{0}^{1} G(s, s) g(s, 1,1) \mathrm{d} s$ are convergent. $\max \left\{r_{1}, r_{3}\right\}<\sqrt[m-1]{\frac{1}{\lambda n}}, m>1$, where

$$
\begin{gathered}
n=\max \left\{r_{2}, r_{4}\right\}, m=\max \left\{\lambda_{1}+\lambda_{2}, \lambda_{3}+\lambda_{4}\right\}, \\
p_{+}(s)=\max \{p(s), 0\}, \quad p_{-}(s)=\max \{-p(s), 0\}, \\
q_{+}(s)=\max \{q(s), 0\}, \quad q_{-}(s)=\max \{-q(s), 0\} .
\end{gathered}
$$

If $(x, y) \in\left(C^{2}[0,1] \cap C^{4}(0,1)\right) \times\left(C^{2}[0,1] \cap C^{4}(0,1)\right)$ satisfies (1.1), (1.2) and $x(t)>0, y(t)>0, \forall t \in(0,1)$, then we call that $(x, y)$ is a positive solution of BVP (1.1), (1.2).

Let

$$
\begin{aligned}
& w_{1}(t)=\int_{0}^{1} G(t, \xi) \mathrm{d} \xi \int_{0}^{1} G(\xi, s) p_{-}(s) \mathrm{d} s, \\
& w_{2}(t)=\int_{0}^{1} G(t, \xi) \mathrm{d} \xi \int_{0}^{1} G(\xi, s) q_{-}(s) \mathrm{d} s,
\end{aligned}
$$

By $\left(H_{2}\right)$ we have

$$
\begin{gathered}
w_{1}(t)=\int_{0}^{1} G(t, \xi) \mathrm{d} \xi \int_{0}^{1} G(\xi, s) p_{-}(s) \mathrm{d} s<+\infty, \\
w_{2}(t)=\int_{0}^{1} G(t, \xi) \mathrm{d} \xi \int_{0}^{1} G(\xi, s) q_{-}(s) \mathrm{d} s<+\infty .
\end{gathered}
$$

By direct computation, we know that $w_{1}(t)$ and $w_{2}(t)$ are positive solutions of the following BVP:

$$
\left\{\begin{array}{l}
u^{(4)}(t)=p_{-}(t), \quad 0<t<1, \\
u(0)=u(1)=u^{\prime \prime}(0)=u^{\prime \prime}(1)=0,
\end{array}\right.
$$

and

$$
\left\{\begin{array}{l}
-v^{(4)}(t)=q_{-}(t), \quad 0<t<1, \\
v(0)=v(1)=v^{\prime \prime}(0)=v^{\prime \prime}(1)=0,
\end{array}\right.
$$

respectively.

We consider the following ordinary differential system

$$
\left\{\begin{array}{c}
x^{(4)}(t)=\lambda f\left(t,\left[x(t)-w_{1}(t)\right]^{*}\right. \\
\left.\left[y(t)-w_{2}(t)\right]^{*}\right)+p_{+}(t) \\
y^{(4)}(t)=\lambda g\left(t,\left[x(t)-w_{1}(t)\right]^{*}\right. \\
\left.\left[y(t)-w_{2}(t)\right]^{*}\right)+q_{+}(t) \\
x(0)=x(1)=x^{\prime \prime}(0)=x^{\prime \prime}(1)=y(0) \\
=y(1)=y^{\prime \prime}(0)=y^{\prime \prime}(1)=0
\end{array}\right.
$$

It is known that $(x, y) \in\left(C^{2}[0,1] \cap C^{4}(0,1)\right) \times\left(C^{2}[0,1]\right.$

$\left.\cap C^{4}(0,1)\right)$ is a solution of system (2.3) if and only if $(x, y) \in C[0,1] \times C[0,1]$ is a solution of the following nonlinear integral equations system

$$
\left\{\begin{aligned}
x(t)= & \int_{0}^{1} G(t, \xi) \mathrm{d} \xi \int_{0}^{1} G(\xi, s)[\lambda f(s,[x(s)- \\
& \left.\left.\left.w_{1}(s)\right]^{*},\left[y(s)-w_{2}(s)\right]^{*}\right)+p_{+}(s)\right] \mathrm{d} s, \\
y(t)= & \int_{0}^{1} G(t, \xi) \mathrm{d} \xi \int_{0}^{1} G(\xi, \mathrm{s})[\lambda g(s,[x(s)- \\
& \left.\left.\left.w_{1}(s)\right]^{*},\left[y(s)-w_{2}(s)\right]^{*}\right)+q_{+}(s)\right] \mathrm{d} s,
\end{aligned}\right.
$$

Let

$$
\begin{aligned}
A(x, y)(t)= & \int_{0}^{1} G(t, \xi) \mathrm{d} \xi \int_{0}^{1} G(\xi, \mathrm{s})[\lambda f(s, \\
& {\left.\left[x(s)-w_{1}(s)\right]^{*},\left[y(s)-w_{2}(s)\right]^{*}\right) } \\
& \left.+p_{+}(s)\right] \mathrm{d} s, \\
B(x, y)(t)= & \int_{0}^{1} G(t, \xi) \mathrm{d} \xi \int_{0}^{1} G(\xi, \mathrm{s})[\lambda g(s, \\
& {\left.\left[x(s)-w_{1}(s)\right]^{*},\left[y(s)-w_{2}(s)\right]^{*}\right) } \\
& \left.+q_{+}(s)\right] \mathrm{d} s,
\end{aligned}
$$

Define a nonlinear integral operator $F: X \rightarrow X$, by $F(x, y)=(A(x, y), B(x, y))$. Thus, system (2.4) is equivalent to the fixed point equation $F(x, y)=(x, y)$ in the Banach space $X=C([0,1] ; R) \times C([0,1] ; R)$.

The proof of main results will be based on the following lemmas.

Lemma 2.1. Let $\Omega_{1}$ and $\Omega_{2}$ be two bounded open sets in Banach space $E$ such that $\theta \in \Omega_{1}, \bar{\Omega}_{1} \subset \Omega_{2}$. A : $P \cap\left(\bar{\Omega}_{2} \backslash \Omega_{1}\right) \rightarrow P$ is a completely continuous operator. Suppose that one of the two conditions holds 
i. $\|A u\| \leq\|u\|, \forall u \in P \bigcap \partial \Omega_{1} ;\|A u\| \geq\|u\|, \forall u \in P \bigcap \partial \Omega_{2}$.

ii. $\|A u\| \geq\|u\|, \forall u \in P \cap \partial \Omega_{1} ; \quad\|A u\| \leq\|u\|, \forall u \in P \bigcap \partial \Omega_{2}$.

Then $A$ have a fixed point in $P \cap\left(\bar{\Omega}_{2} \backslash \Omega_{1}\right)$.

Lemma 2.2. If $f(t, x, y)$ and $g(t, x, y)$ satisfy $\left(H_{1}\right)$, $\left(H_{2}\right)$, then $f$ and $g$ are nondecreasing in both $x, y \in[0,+\infty)$ for any fixed $t \in(0,1)$, we have

$$
\begin{array}{ll}
\lim _{\substack{x \rightarrow+\infty \\
y \geq 0}} \frac{f(t, x, y)}{|(x, y)|}=+\infty, & \lim _{\substack{y \rightarrow+\infty \\
x \geq 0}} \frac{f(t, x, y)}{|(x, y)|}=+\infty, \\
\lim _{\substack{x \rightarrow+\infty \\
y \geq 0}} \frac{g(t, x, y)}{|(x, y)|}=+\infty, & \lim _{\substack{y \rightarrow+\infty \\
x \geq 0}} \frac{g(t, x, y)}{|(x, y)|}=+\infty,
\end{array}
$$

where $|(x, y)|=|x|+|y|$.

Proof. For any fixed $t \in(0,1), 0<x_{1}<x_{2}$, it follows from $\left(H_{1}\right)$ that

$$
\begin{aligned}
f\left(t, x_{1}, y\right) & =f\left(t, \frac{x_{1}}{x_{2}} x_{2}, y\right) \\
& \leq\left(\frac{x_{1}}{x_{2}}\right)^{\mu_{1}} f\left(t, x_{2}, y\right) \leq f\left(t, x_{2}, y\right),
\end{aligned}
$$

Thus, $f(t, x, y)$ is nondecreasing in $x \in[0,+\infty)$.

In the same way, we know $f(t, x, y)$ is nondecreasing in $y \in[0,+\infty) . g(t, x, y)$ are nondecreasing in $x, y \in[0,+\infty)$ for any fixed $t \in(0,1)$.

On the other hand, for any $x>1, y \geq 0$, it follows from $\left(H_{2}\right)$, when $x \geq y$, we have

$$
\begin{aligned}
\frac{f(t, x, y)}{|(x, y)|} & =\frac{f(t, x, y)}{|x|+|y|} \\
& \geq \frac{x^{\mu_{1}} f(t, 1, y)}{2 x} \geq \frac{1}{2} x^{\mu_{1}-1} f(t, 1,0)>0,
\end{aligned}
$$

when $x<y$, we have $y>1$ and

$$
\begin{aligned}
\frac{f(t, x, y)}{|(x, y)|} & =\frac{f(t, x, y)}{|x|+|y|} \\
& \geq \frac{y^{\mu_{2}} f(t, x, 1)}{2 y} \geq \frac{1}{2} y^{\mu_{2}-1} f(t, 0,1)>0,
\end{aligned}
$$

Therefore, we obtain $\lim _{\substack{x \rightarrow+\infty \\ y \geq 0}} \frac{f(t, x, y)}{|(x, y)|}=+\infty$.

In the same way, we have

$$
\begin{gathered}
\lim _{\substack{y \rightarrow+\infty \\
x \geq 0}} \frac{f(t, x, y)}{|(x, y)|}=+\infty, \quad \lim _{\substack{x \rightarrow+\infty \\
y \geq 0}} \frac{g(t, x, y)}{|(x, y)|}=+\infty, \\
\lim _{\substack{y \rightarrow+\infty \\
x \geq 0}} \frac{g(t, x, y)}{|(x, y)|}=+\infty .
\end{gathered}
$$

Lemma 2.3. ${ }^{[10]}$ If $(u, v)$ with $u(t)>w_{1}(t), v(t)>w_{2}(t)$ for any $t \in(0,1)$ is a positive solution of system $(2.4)$, then $\left(u-w_{1}, v-w_{2}\right)$ is a positive solution of the semipositone singular differential system (1.1), (1.2).

Lemma 2.4. Suppose that $\left(H_{1}\right),\left(H_{2}\right)$ hold, Then $F$ : $P \rightarrow P$ is a completely continuous operator.

Proof. For any fixed $(x, y) \in P$, choose $0<a, b<1$, such that $a\|x\|<1, b\|y\|<1$, then

$$
\begin{aligned}
& a\left[x(t)-w_{1}(t)\right]^{*} \leq a x(t) \leq a\|x\|<1, \\
& b\left[y(t)-w_{2}(t)\right]^{*} \leq b y(t) \leq b\|y\|<1 .
\end{aligned}
$$

Hence, by $\left(H_{1}\right)$ we have

$$
\begin{aligned}
& f\left(t,\left[x(t)-w_{1}(t)\right]^{*},\left[y(t)-w_{2}(t)\right]^{*}\right) \\
\leq & a^{\mu_{1}-\lambda_{1}} b^{\mu_{2}-\lambda_{2}}\|x\|^{\mu_{1}}\|y\|^{\mu_{2}} f(t, 1,1) .
\end{aligned}
$$

Consequently, for any $t \in[0,1]$, we have

$$
\begin{aligned}
A(x, y)(t) \leq & \frac{1}{4} \int_{0}^{1} G(s, s)\left[\lambda f \left(s,\left[x(s)-w_{1}(s)\right]^{*},\right.\right. \\
& {\left.\left.\left[y(s)-w_{2}(s)\right]^{*}\right)+p_{+}(s)\right] \mathrm{d} s } \\
\leq & \frac{1}{4} \int_{0}^{1} G(s, s)\left[\lambda a^{\mu_{1}-\lambda_{1}} b^{\mu_{2}-\lambda_{2}}\|x\|^{\mu_{1}}\|y\|^{\mu_{2} .} .\right. \\
& \left.f(s, 1,1)+p_{+}(s)\right] \mathrm{d} s \\
\leq & \frac{1}{4}\left(\lambda a^{\mu_{1}-\lambda_{1}} b^{\mu_{2}-\lambda_{2}}\|x\|^{\mu_{1}}\|y\|^{\mu_{2}}+1\right) . \\
& \int_{0}^{1} G(s, s)\left[f(s, 1,1)+p_{+}(s)\right] \mathrm{d} s<+\infty .
\end{aligned}
$$

In the same way, we also have $B(x, y)(t)<+\infty$. Thus $F$ : $P \rightarrow X$ is well defined.

There exists a $t_{1} \in[0,1]$ such that

$$
A(x, y)\left(t_{1}\right)=\|A(x, y)\| .
$$

Since $G(t, s) \geq t(1-t) G\left(t_{1}, s\right), \forall t, s \in[0,1]$, then, we have

$$
\begin{aligned}
A(x, y)(t) \geq & t(1-t) \int_{0}^{1} G\left(t_{1}, \xi\right) \mathrm{d} \xi \int_{0}^{1} G(\xi, s) . \\
& {\left[\lambda f\left(s,\left[x(s)-w_{1}(s)\right]^{*},\left[y(s)-w_{2}(s)\right]^{*}\right)\right.} \\
& \left.+p_{+}(s)\right] \mathrm{d} s \\
\geq & A(x, y)\left(t_{1}\right) t(1-t)=t(1-t)\|A(x, y)\| .
\end{aligned}
$$

In the same way, there exists a $t_{2} \in[0,1]$ such that

$$
B(x, y)\left(t_{2}\right)=\|B(x, y)\| .
$$

Using the same way as the above proof, we also have

$$
B(x, y)(t) \geq t(1-t)\|B(x, y)\| .
$$

Then, $F(P) \subset P$.

By proceeding as for the proof of Lemma 2.4 in [10], $F$ : $P \rightarrow P$ is a completely continuous operator. 


\section{Main Result}

Theorem 3.1. Suppose that $\left(H_{1}\right),\left(H_{2}\right)$ are satisfied, then two-point boundary value problem $(1.1),(1.2)$ has at least a $\left(C^{2}[0,1] \cap C^{4}(0,1)\right) \times\left(C^{2}[0,1] \cap C^{4}(0,1)\right)$ positive solution.

Proof. Choose $R_{1}$ such that

$$
\max \left\{r_{1}, r_{3}, 1\right\}<R_{1}<\sqrt[m-1]{\frac{1}{\lambda n}}
$$

Let

$$
\Omega_{R_{1}}=\left\{(x, y) \in P \mid\|x\|<R_{1},\|y\|<R_{1}\right\},
$$

$\|z\|=\max \{\|x\|,\|y\|\}$, then $\forall(x, y) \in P \bigcap \partial \Omega_{R_{1}}$, we can obtain $\|z\|>1$ and

$$
\begin{aligned}
& |F(x, y)(t)|=|A(x, y)(t)|+|B(x, y)(t)| \\
\leq & \left|\int_{0}^{1} G(t, \xi) d \xi \int_{0}^{1} G(\xi, s)\left[\lambda f(s, x(s), y(s))+p_{+}(s)\right] \mathrm{d} s\right| \\
+ & \left|\int_{0}^{1} G(t, \xi) d \xi \int_{0}^{1} G(\xi, s)\left[\lambda g(s, x(s), y(s))+q_{+}(s)\right] \mathrm{d} s\right| \\
\leq & \frac{1}{4}\left|\int_{0}^{1} G(\xi, s)\left[\lambda\|z\|^{\lambda_{1}+\lambda_{2}} f(s, 1,1)+p_{+}(s)\right] \mathrm{d} s\right| \\
& +\frac{1}{4}\left|\int_{0}^{1} G(\xi, s)\left[\lambda\|z\|^{\lambda_{3}+\lambda_{4}} g(s, 1,1)+q_{+}(s)\right] \mathrm{d} s\right| \\
\leq & \frac{1}{4}\left(\lambda\|z\|^{\lambda_{1}+\lambda_{2}}+1\right) \int_{0}^{1} G(s, s)\left[f(s, 1,1)+p_{+}(s)\right] \mathrm{d} s \\
& +\frac{1}{4}\left(\lambda\|z\|^{\lambda_{3}+\lambda_{4}}+1\right) \int_{0}^{1} G(s, s)\left[g(s, 1,1)+q_{+}(s)\right] \mathrm{d} s \\
\leq & \frac{1}{4}\left(\lambda\|z\|^{m}+1\right) r_{2}+\frac{1}{4}\left(\lambda\|z\|^{m}+1\right) r_{4} \\
\leq & \frac{1}{4}\left(\lambda r_{2} R_{1}^{m-1} R_{1}+R_{1}\right)+\frac{1}{4}\left(\lambda r_{4} R_{1}^{m-1} R_{1}+R_{1}\right) \\
\leq & \frac{1}{4}\left(\lambda r_{2} \frac{1}{\lambda n} R_{1}+R_{1}\right)+\frac{1}{4}\left(\lambda r_{4} \frac{1}{\lambda n} R_{1}+R_{1}\right) \\
\leq & \frac{1}{4}\left(2 R_{1}\right)+\frac{1}{4}\left(2 R_{1}\right)=R_{1},
\end{aligned}
$$

As a consequence,

$\|F(x, y)\| \leq\|x\|+\|y\|=\|(x, y)\|, \forall(x, y) \in P \cap \partial \Omega_{R_{1}}$.

We choose constant $L, M$ such that

$$
\begin{gathered}
M>\frac{4}{3}\left[\lambda \alpha^{2}(1-\beta)^{2}(\beta-\alpha)\left(\min _{t \in[\alpha, \beta]} \int_{\alpha}^{\beta} G(t, \xi) \mathrm{d} \xi\right)\right]^{-1}, \\
L>R_{1} .
\end{gathered}
$$

From Lemma 2.2, when $x \geq L, y \geq 0$, we have

$$
\frac{f(t, x, y)}{|(x, y)|} \geq M \text {, }
$$

that is, $f(t, x, y) \geq M|(x, y)|$.

In the same way, when $x \geq L, y \geq 0$, we have $g(t, x, y)$ $\geq M|(x, y)|$, (where $[\alpha, \beta] \in(0,1))$.
Choose $R_{2}>\frac{4 L}{3 \alpha(1-\beta)}$, then $R_{2}>L>R_{1}$, thus $\frac{R_{1}}{R_{2}}<1$. Let

$$
\Omega_{R_{2}}=\left\{(x, y) \in P \mid\|x\|<R_{2},\|y\|<R_{2}\right\},
$$

Then $\forall(x, y) \in P \bigcap \partial \Omega_{R_{2}},\|x\|$ and $\|y\|$ have at least a $R_{2}$. Noticing that

$$
\begin{aligned}
w_{1}(t) & =\int_{0}^{1} G(t, \xi) \mathrm{d} \xi \int_{0}^{1} G(\xi, s) p_{-}(s) \mathrm{d} s \\
& \leq \frac{1}{4} t(1-t) \int_{0}^{1} p_{-}(s) \mathrm{d} s \leq \frac{1}{4} t(1-t) r_{1} .
\end{aligned}
$$

In the same way, $w_{2}(t) \leq \frac{1}{4} t(1-t) r_{3}$.

1. Both $\|x\|$ and $\|y\|$ are $R_{2}$,

$$
\begin{aligned}
& x(t)-w_{1}(t) \geq x(t)-\frac{1}{4} t(1-t) r_{1} \\
\geq & x(t)-\frac{1}{4} t(1-t) R_{1} \geq x(t)-\frac{x(t)}{4\|x\|} R_{1} \\
\geq & x(t)-\frac{R_{1}}{4 R_{2}} x(t) \geq \frac{3}{4} t(1-t)\|x\| \\
\geq & \frac{3}{4} \alpha(1-\beta) R_{2} \geq L .
\end{aligned}
$$

In the same way, $y(t)-w_{2}(t) \geq L$.

2. One of $\|x\|$ and $\|y\|$ is $R_{2}$, without loss of the generality, let $\|x\|=R_{2}$, we have $x(t)-w_{1}(t) \geq L$, and $\left[y(t)-w_{2}(t)\right]^{*} \geq 0$. This together with Lemma 2.2 yields

$$
\begin{aligned}
& |F(x, y)(t)|=|A(x, y)(t)|+|B(x, y)(t)| \\
\geq & \int_{\alpha}^{\beta} G(t, \xi) d \xi \int_{\alpha}^{\beta} G(\xi, s) \lambda M \cdot \\
& \left|\left(x(s)-w_{1}(s),\left[y(s)-w_{2}(s)\right]^{*}\right)\right| \mathrm{ds} \\
& +\int_{\alpha}^{\beta} G(t, \xi) d \xi \int_{\alpha}^{\beta} G(\xi, s) \lambda M \cdot \\
& \left|\left(x(s)-w_{1}(s),\left[y(s)-w_{2}(s)\right]^{*}\right)\right| \mathrm{ds} \\
\geq & \int_{\alpha}^{\beta} G(t, \xi) d \xi \int_{\alpha}^{\beta} G(\xi, s) \lambda M\left|x(s)-w_{1}(s)\right| \mathrm{d} s \\
& +\int_{\alpha}^{\beta} G(t, \xi) d \xi \int_{\alpha}^{\beta} G(\xi, s) \lambda M\left|x(s)-w_{1}(s)\right| \mathrm{d} s \\
\geq & \frac{3}{4} \lambda M \alpha^{2}(1-\beta)^{2} R_{2}(\beta-\alpha) \int_{\alpha}^{\beta} G(t, \xi) \mathrm{d} \xi \\
& +\frac{3}{4} \lambda M \alpha^{2}(1-\beta)^{2} R_{2}(\beta-\alpha) \int_{\alpha}^{\beta} G(t, \xi) \mathrm{d} \xi \\
\geq & R_{2}+R_{2}, \forall t \in[\alpha, \beta], \forall(x, y) \in P \cap \partial \Omega_{R_{2}} .
\end{aligned}
$$

Thus,

$$
\|F(x, y)\| \geq\|x\|+\|y\|=\|(x, y)\|, \forall(x, y) \in P \bigcap \partial \Omega_{R_{2}} .
$$


As a consequence, by lemma $2.1 F$ has at least one fixed point $\left(x_{0}, y_{0}\right) \in P \cap\left(\bar{\Omega}_{R_{2}} \backslash \Omega_{R_{1}}\right)$ with $R_{1} \leq\left\|x_{0}\right\| \leq R_{2}$, and $R_{1} \leq\left\|y_{0}\right\| \leq R_{2}$.

Hence, for any $t \in(0,1)$, we have

$$
\begin{aligned}
& x_{0}(t)-w_{1}(t) \\
\geq & \left\|x_{0}\right\| t(1-t)-\int_{0}^{1} G(t, \xi) \mathrm{d} \xi \int_{0}^{1} G(\xi, s) p_{-}(s) \mathrm{d} s \\
\geq & \left\|x_{0}\right\| t(1-t)-\frac{1}{4} t(1-t) r_{1}>0, \quad \forall t \in(0,1) .
\end{aligned}
$$

In the same way, we have

$$
y_{0}(t)-w_{2}(t)>0, \forall t \in(0,1) .
$$

It follows from lemma 2.3 that $\left(x_{0}-w_{1}, y_{0}-w_{2}\right)$ is one positive solution of the boundary value problem (1.1), (1.2) in $\left(C^{2}[0,1] \cap C^{4}(0,1)\right) \times\left(C^{2}[0,1] \cap C^{4}(0,1)\right)$.

\section{References}

[1] Guo Dajun, Lakshmikantham V, Nonlinear problems in abstract cones[M],San Diego: Academic Press,1998, 24-56.

[2] Anuradha V, Hai D, Shivaji R, Existence results for superlin- ear semipositone BVP's[J], Pro Amer Math Soc, 1996, 124(3): 747-763.

[3] Agarwal R P, O'Regan D, A note on existence of nonnegative solutions to singular semipositone problems[J], Nonlinear Anal, 1999, 36(5): 615-622.

[4] Xu Xian, Positive solutions for singular semipositone boundary value problems[J], J Math Anal Appl, 2002,273(2): 480-491.

[5] Zhang Xinguang, Liu Lishan, Positive solutions of superlinear semipositone singular Dirichlet boundary value problems[J], J Math Anal Appl, 2006, 316(2): 525-537.

[6] Zhang Xinguang, Zhao Zenqin, Positive solution of fourth order singular semipositone boundary value problem[J], J Systems Sci Math Sci, 2006, 26(5): 553-560.

[7] Fink A M, Gatica J A, Positive solutions of second order systems of boundary value problems[J], J Math Anal Appl, 1993, 180(1): 93-108.

[8] Agarwal R P, O'Regan D, A coupled system of boundary value problems[J], Appl Anal, 1998, 69: 381-385.

[9] Xu Xian, Positive solutions for singular semipositone threepoint systems[J], Nonlinear Anal, 2007, 66: 791-805.

[10] Liu Lishan, Zhang Xinguang, Wu Yonghong, On existence of positive solutions of a two-point boundary problem for a nonlinear singular semipositone system[J], Appl Math Comput, 2007, 192: 223-232. 